\title{
Two new species of Euscorpius from Euboea Island, Greece (Scorpiones: Euscorpiidae)
}

\author{
Ава новых вида Еuscorpius с острова Эвбея, Грещия \\ (Scorpiones: Euscorpiidae)
}

\author{
Victor Fet ${ }^{1}$, Michael E. Soleglad ${ }^{2}$, Aristeidis Parmakelis ${ }^{3}$, \\ Panayiota Kotsakiozi ${ }^{4}$ \& Iasmi Stathi ${ }^{5}$ \\ В.Я. Фет ${ }^{1}$, М.Ю. СолеглаА ${ }^{2}$, А. Пармакелис ${ }^{3}$, \\ П. Котсакиози ${ }^{4}$, Я. Стати
}

\footnotetext{
${ }^{1}$ Department of Biological Sciences, Marshall University, Huntington, West Virginia 25755-2510, USA. E-mail: fet@marshall.edu (Corresponding author).

232255 Safflower St., Winchester, California 92596, USA. E-mail: m-soleglad@znet.com

${ }^{3}$ Department of Ecology and Taxonomy, Faculty of Biology, University of Athens, Athens, Greece. E-mail: parmakel@nhmc.uoc.gr

${ }^{4}$ Department of Human and Animal Physiology, Faculty of Biology, University of Athens, Athens, Greece. E-mail: pkotsakiozi@biol.uoa.gr

${ }^{5}$ Natural History Museum of Crete, University of Crete, Heraklion, Crete, Greece. E-mail: iasmi@nhmc.uoc.gr
}

KEY WORDS: scorpions, Euscorpius, Greece, Euboea, new species, zoogeography.

КЛЮЧЕВЫЕ СЛОВА: скорпионы, Euscorpius, Греция, Эвбея, новые виды, зоогеография.

ABSTRACT. We describe two new species of the genus Euscorpius from Euboea Island, Greece: E. birulai sp.n. (Agia Triada Cave) and E. mylonasi sp.n. (Mt. Dirfi). The cave species E. birulai sp.n. is morphologically close to E. koschewnikowi Birula, 1900 from Mt. Athos, Greece. Species-level divergence of E. mylonasi sp.n. is also confirmed by multiple DNA markers. According to DNA data, an undescribed population from the nearby Skyros Island is closely related to E. mylonasi sp.n.

РЕЗЮМЕ. Описаны два новых вида рода Euscorpius с острова Эвбея, Греция: E. birulai sp.n. (пещера Агиа Триада) и E. mylonasi sp.n. (горы Дирфи). Пещерный вид E. birulai sp.n. близок к E. koschewnikowi Birula, 1900 с горы Афон, Греция. Дивергенция на уровне вида для mylonasi sp.n. подтверждена несколькими ДНК-маркерами. Согласно данным ДНК, неописанная популяция с соседнего острова Скирос близка к E. mylonasi sp.n.

\section{Introduction}

The taxonomy of the widespread Mediterranean genus Euscorpius Thorell, 1876 (Euscorpiidae) is insufficiently studied. In Greece total number of confirmed species of the genus was recently raised to 12 : E. avcii Tropea, Yağmur, Koç, Yesilyurt et Rossi, 2012; E. candiota Birula, 1903; E. corcyraeus Tropea et Rossi, 2012; E. erymanthius Tropea, Fet, Parmakelis,
Kotsakiozi et Stathi, 2013; E. hadzii Di Caporiacco, 1950; E. italicus (Herbst, 1800); E. koschewnikowi Birula, 1900; E. kritscheri Fet, Soleglad, Parmakelis, Kotsakiozi et Stathi, 2013; E. naupliensis (C.L. Koch, 1837); E. ossae Di Caporiacco, 1950; E. scaber Birula, 1900; and E. sicanus (C.L. Koch, 1837)) [Fet et al., 2013a]. Our data [Parmakelis et al., 2013; unpublished data] indicate that a number of undescribed specieslevel forms of Euscorpius, including island endemics, are also present in Greece.

As a part of an ongoing revisionary study, we describe here two new species from the second largest (after Crete) Greek island of Euboea (Gr. Åýâiéá, also spelled Evvoia, Evvia, Evia, or Euboia) in the western Aegean Sea. Euscorpius populations from Euboea were listed under " $E$. carpathicus" in the recent literature [Kinzelbach, 1975; Kritscher, 1993; Stathi \& Mylonas, 2001] but specimens from this island were never analyzed. Validity of E. mylonasi sp.n. described herein from Euboea was supported by our molecular (DNA) phylogenetic study of Euscorpius populations across Greece [Parmakelis et al., 2013]. In addition, we discuss an undescribed Euscorpius population from a nearby Skyros Island, which, according to DNA data, is closely related to E. mylonasi sp.n.

\section{Material and methods}

All DNA work was performed in the University of Athens by P.K. and A.P. For details on molecular 
and phylogenetic analysis, see Parmakelis et al. [2013].

The specimens examined are preserved in the following collections: MCSNG - Museo Civico di Storia Naturale "Giacomo Doria", Genoa, Italy; MRSN Museo Regionale di Scienze Naturali di Torino, Torino, Italy; NHMC — Natural History Museum of Crete, University of Crete, Heraklion, Crete, Greece; NHMW - Naturhistorisches Museum Wien, Vienna, Austria; NMNHS - National Museum of Natural History, Sofia, Bulgaria; PMGPC — personal collection of Pier Mauro Giachino, Torino, Italy; ZMHB - Museum für Naturkunde, Humboldt-Universität zu Berlin, Germany). Nomenclature for reporting DNA sequences from type ("geneseq-2") and non-type ("geneseq3") specimens follows Chakrabarty et al. [2013].

\section{Results}

Genus Euscorpius Thorell, 1876

Subgenus incertae sedis

Euscorpius birulai Fet, Soleglad, Parmakelis, Kotsakiozi et Stathi, sp.n.

Figs 2-16; Tables 1-2.

TYPE MATERIAL: Holotype OT, GREECE, Central Greece: Euboea Island, Karistos, Agia Triada Cave, $38^{\circ} 02^{\prime} 35.24^{\prime \prime} \mathrm{N}, 24^{\circ}$ 25'06.13"E, 200 m, 2 January 2003, leg. P. Beron (NMNHS 266). Paratypes: same label as holotype, $1 \mathrm{O}^{7}, 2 \mathrm{O}^{7} \mathrm{O}^{7}$ subad., $1 \mathrm{O}^{7} \mathrm{im} ., 3$ of $\mathrm{im}$. (NMNHS 266); same locality, 9 June 2002, leg. P.M. Giachino \& D. Vailati, $20^{7} O^{7}$ (MHNSG, PMGC Sc61).

DIAGNOSIS. A brown, slender, medium-sized species (32-40 $\mathrm{mm}$ for adult males). Pectinal teeth 7 in males, patellar trichobothria et $=5-6(6)$ and $v=8$; est - est $_{4}$ form a straight line. Median indentation present on carapace anterior edge; dorsal patellar spur (DPS) considerably long and sharp, providing a DPS ratio of 1.0; all metasomal segments are longer than wide in adult males; metasomal ventromedian carina is absent on all segments.

ETYMOLOGY. The species name is a patronym honoring Dr. Alexei Andreevich Byalynitskyi-Birula (1864-1937), a famous Russian zoologist, prominent polar researcher, and one of the greatest scorpion systematists of all times [see e.g. Birula, 1900, 1917a, b]. Birula was a Director of the Zoological Institute in Leningrad (ZISP; former Imperial Zoological Museum, now Zoological Institute, Russian Academy of Sciences, St. Petersburg, Russia) when he was demoted in 1929, arrested in 1930 and sent to concentration camps for three years. His scorpion collection remains largely intact in ZISP.

DISTRIBUTION. Known only from Agia Triada Cave, Karistos, Euboea Island, Greece. See map in Fig. 1.

DESCRIPTION. Male. The following description is based primarily on the holotype male from Agia Triada Cave, Karistos, Euboea Island, Greece, with additional information derived from a paratype male. Measurements of the holotype and paratype males are presented in Table 1. See Fig. 2 for dorsal and ventral views of the male holotype.

Coloration. Carapace and pedipalps dark brown; tergites, metasoma, telson, legs sternites, and chelicerae brown; leg coxae and sternum dark brown; genital operculum, pectines, basal piece, and yellow. No patterns present.

Carapace (Figs 3-14, paratype males). Anterior edge with a median indentation; smooth and lustrous, lacking any indication of carinae. Two lateral eyes. Median eyes and tubercle small in size, positioned anterior of middle with the following length and width ratios: 0.403 (anterior edge to medium tubercle middle / carapace length) and 0.145 (width of median tubercle including eyes / width of carapace at that point).

Mesosoma. Tergites I-VII essentially smooth; tergite VII lacking lateral and median carinal pairs. Sternites III-VII smooth and lustrous; VII lacking lateral and median carinae. Stigmata very small, narrow elliptical.

Metasoma (Fig. 9). Segment I longer than wide in ratio 1.028. Segments I-IV: dorsal, dorsolateral, and lateral carinae are obsolete; ventrolateral carinae obsolete on I-II, vestigially present and smooth on III-IV; single ventromedian carina obsolete. Segment V: dorsolateral and lateral carinae obsolete, ventrolateral vestigially present and smooth, and ventromedian carinae obsolete. Anal arch with approximately 16 small pigmented granules. Intercarinal areas smooth.

Telson (Fig. 6). Vesicle swollen and elongated, with short highly curved aculeus. Vesicle essentially void of granules, very lustrous. Vesicular tabs smooth.

Pectines (Fig. 7). Medium-developed segments exhibiting length / width ratio 1.974 (length taken at anterior lamellae / width at widest point including teeth). Sclerite construction complex, three anterior lamellae and four middle lamella; fulcra of medium development. Teeth number 7/7. Sensory areas developed along distal aspect on all teeth, including basal tooth. Basal piece large, with subtle shallow indentation along anterior edge, length / width ratio 0.457 .

Chelicerae (Fig. 8). Movable finger dorsal edge with two small subdistal $(s d)$ denticles; ventral edge smooth; serrula not visible. Ventral distal denticle $(v d)$ conspicuously longer than dorsal $(d d)$. Fixed finger with four denticles, median $(m)$ and basal $(b)$ denticles conjoined on common trunk; no ventral accessory denticles present.

Pedipalps (Figs 10-11, paratype male Figs 12, 15). Well-developed chelae, moderately carinated, strong scalloping on chelal fingers. Femur: Dorsointernal and ventrointernal carinae heavily serrated, dorsoexternal lightly serrated, and ventroexternal rounded with scattered granulation, heaviest at base. Dorsal surface covered with small granules proximally, ventral surface scattered with small granules, internal and external surfaces rough with a row of serrated granules. Patella: Dorsointernal and ventrointernal carinae heavily crenu- 


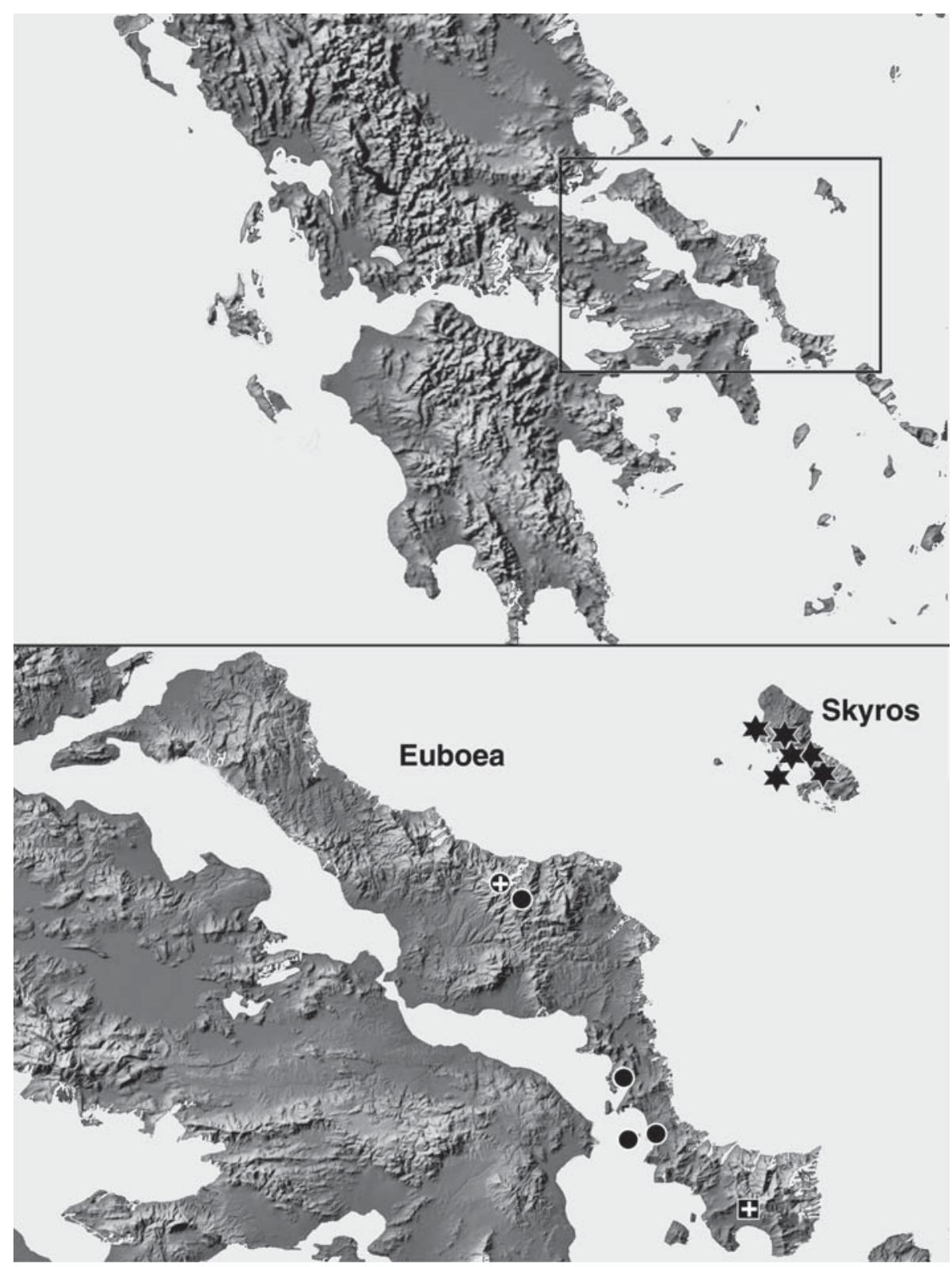

Fig. 1. Map showing type localities and known distribution of Euscorpius mylonasi sp.n. (circle icons), E. birulai sp.n. (square icon), and a population of Euscorpius cf. mylonasi from Skyros Island (star icons). Type localities are marked with '+'. Map on bottom shows detail of the species' type localities and distribution. Map on top shows a larger portion of Greece proper with the detailed map indicated by a yellow box.

Рис. 1. Карта типовых местонахождений и распространения Euscorpius mylonasi sp.n. (круг) и E. birulai sp.n. (квадрат) на острове Эвбея, и распространение Euscorpius cf. mylonasi c о. Скирос (звездочка). Типовые местонахождения обозначены знаком '+'. На врезке - подробная карта распространения.

lated, dorsoexternal irregularly granulate, ventroexternal moderately crenulated, and exteromedian carina developed lined with medium sized granules. Dorsal and ventral surfaces smooth; external rough; internal surface smooth with well-developed, long and pointed DPS and near obsolete VPS (single small granule). Chelal carinae: comply with the "10-carinae configuration". Digital $(D 1)$ carina strong, slight traces of lowprofile granulation; sub-digital (D2) essentially obso- lete, represented by one granule; dorsosecondary $(D 3)$ near obsolete, area quite flat, presented by one large basal granule; dorsomarginal (D4) rounded, with medium-sized granules; dorsointernal (D5) highly rounded and covered with granules; ventroexternal (VI) strong, evenly granulated, curving to external condyle of movable finger; ventromedian ( $V 2)$ obsolete; ventrointernal $(V 3)$ rounded with scattered granulation; external $(E)$ irregularly developed with scattered granulation on 
Table 1. Morphometrics (mm) of Euscorpius mylonasi sp.n. and E. birulai sp.n. Таблица 1. Измерения (мм) of Euscorpius mylonasi sp.n. и E. birulai sp.n. .

\begin{tabular}{|c|c|c|c|c|c|}
\hline & \multicolumn{3}{|c|}{ Euscorpius mylonasi } & \multirow{2}{*}{\multicolumn{2}{|c|}{$\begin{array}{c}\text { Euscorpius birulai } \\
\text { Agia Triada Cave, } \\
\text { Karistos, } \\
\text { Euboea Island, } \\
\text { Greece } \\
\end{array}$}} \\
\hline & \multicolumn{2}{|c|}{$\begin{array}{c}\text { Mt. Dirfi, } \\
\text { Euboea Island, Greece }\end{array}$} & \multirow{2}{*}{$\begin{array}{c}\text { Agios Andreas } \\
\text { islet, Euboea } \\
\text { Island, } \\
\text { Greece } \\
\text { Male } \\
\text { Paratype }\end{array}$} & & \\
\hline & $\begin{array}{c}\text { Male } \\
\text { Holotype }\end{array}$ & $\begin{array}{c}\text { Female } \\
\text { Paratype }\end{array}$ & & $\begin{array}{c}\text { Male } \\
\text { Holotype }\end{array}$ & $\begin{array}{c}\text { Male } \\
\text { Paratype }\end{array}$ \\
\hline Total length & 30.80 & 25.95 & 28.20 & 38.95 & 31.55 \\
\hline Carapace length & 4.30 & 3.50 & 3.65 & 5.70 & 4.75 \\
\hline Mesosoma length & 11.65 & 11.00 & 10.10 & 13.50 & 8.65 \\
\hline Metasoma length & 10.95 & 8.35 & 10.45 & 14.30 & 13.00 \\
\hline $\begin{array}{l}\text { Segment I } \\
\text { length/width }\end{array}$ & $1.40 / 1.60$ & $1.10 / 1.40$ & $1.30 / 1.30$ & $1.85 / 1.80$ & $1.65 / 1.55$ \\
\hline $\begin{array}{l}\text { Segment II } \\
\text { length/width }\end{array}$ & $1.65 / 1.40$ & $1.30 / 1.20$ & $1.60 / 1.20$ & $2.15 / 1.45$ & $2.05 / 1.40$ \\
\hline $\begin{array}{l}\text { Segment III } \\
\text { length/width }\end{array}$ & $1.90 / 1.40$ & $1.45 / 1.15$ & $1.80 / 1.15$ & $2.50 / 1.40$ & $2.25 / 1.35$ \\
\hline $\begin{array}{l}\text { Segment IV } \\
\text { length/width }\end{array}$ & $2.20 / 1.30$ & $1.70 / 1.10$ & $2.25 / 1.10$ & $2.80 / 1.30$ & $2.75 / 1.20$ \\
\hline $\begin{array}{l}\text { Segment V } \\
\text { length/width }\end{array}$ & $3.80 / 130$ & $2.80 / 1.05$ & $3.50 / 1.10$ & $5.00 / 1.40$ & $4.30 / 1.30$ \\
\hline Telson length & 3.90 & 3.10 & 4.00 & 5.45 & 5.15 \\
\hline Vesicle length & 3.25 & 2.25 & 3.15 & 4.30 & 4.00 \\
\hline width/depth & $1.65 / 1.60$ & $1.20 / 0.95$ & $1.35 / 1.60$ & $2.25 / 2.40$ & $1.95 / 2.05$ \\
\hline Aculeus length & 0.65 & 0.85 & 0.85 & 1.15 & 1.15 \\
\hline Pedipalp length & 14.50 & 12.10 & 13.65 & 21.50 & 18.85 \\
\hline $\begin{array}{l}\text { Femur } \\
\text { length/width }\end{array}$ & $3.50 / 1.40$ & $3.00 / 1.25$ & $3.35 / 1.25$ & $5.40 / 1.85$ & $4.80 / 1.50$ \\
\hline Patella & & & & & \\
\hline length/width* & $3.65 / 1.45$ & $3.15 / 1.20$ & $3.35 / 1.30$ & $5.30 / 1.60$ & $4.75 / 1.45$ \\
\hline DPS height*** & 0.40 & 0.45 & 0.45 & 1.10 & 0.95 \\
\hline Chela length & 7.35 & 5.95 & 6.95 & 10.80 & 9.30 \\
\hline Palm length & 4.00 & 3.25 & 3.55 & 5.80 & 4.65 \\
\hline width/depth & $2.70 / 3.25$ & $2.10 / 2.25$ & $2.50 / 2.75$ & $3.25 / 3.45$ & $2.80 / 2.90$ \\
\hline Fixed finger length & 3.30 & 2.60 & 2.85 & 4.30 & 4.00 \\
\hline Movable finger length & 4.50 & 3.25 & 3.90 & 5.65 & 5.20 \\
\hline $\begin{array}{l}\text { Sternum } \\
\text { length/width }\end{array}$ & $1.35 / 1.40$ & $1.15 / 1.30$ & $1.40 / 1.50$ & $1.60 / 1.50$ & $1.35 / 1.30$ \\
\hline $\begin{array}{l}\text { Pectines } \\
\text { teeth } \\
\text { middle lamellae }\end{array}$ & $\begin{array}{l}8 / 8 \\
5 / 6\end{array}$ & $\begin{array}{l}8 / 7 \\
5 / 4\end{array}$ & $\begin{array}{l}8 / 8 \\
6 / 5\end{array}$ & $\begin{array}{l}7 / 7 \\
4 / 4\end{array}$ & $\begin{array}{l}7 / 7 \\
4 / 4\end{array}$ \\
\hline
\end{tabular}

*Patella width is the widest distance between the dorsointernal and externomedial carinae.

** DPS height is from tip of spine to dorsointernal carina centered.

*Ширина пателлы измеряется как максимальное расстояние между внутренним дорзальным и внешним медиальным килями. **Высота дорзального шипа пателлы (DPS) измеряется от кончика шипа до внутреннего дорзального киля

mid-distal area. Chelal finger dentition: Median denticle $(M D)$ row groups in straight line; 6/6 IDs fixed finger and 7/7 on movable finger; 6/6 ODs on fixed and movable fingers; 4/4 and 5/5 IADs on fixed and movable fingers, respectively. Trichobothrial patterns (Fig. 15, paratype male): Type $\mathrm{C}$, neobothriotaxic: chela ventral $=4 / 4$; patellar $e b=4 / 4, e b=4 / 4, e s b=2 / 2, e m=$ $4 / 4$, est $=4 / 4$, et $=6 / 6$; patellar ventral $=8 / 8$; est $_{2}-$ est $_{4}$ form a straight line.

Legs (Fig. 13, male paratype). Both pedal spurs present on all legs, lacking spinelets; tibial spurs absent. Tarsus with single row of spinules on ventral surface, terminating distally with two essentially adjacent spinules. Unguicular spine well-developed and pointed.

Hemispermatophore (Fig. 16, male paratype). Right hemispermatophore is $5.60 \mathrm{~mm}$ in length, trunk 2.24, and lamina 3.36. Well-developed truncal flexure. Lamina with a conspicuous basal constriction, terminus tapered and curving towards the exteroventral surface. Median projection with both primary and secondary acuminate processes. The basal and auxiliary lobes and spur are present on the primary acuminate process. The internal projection crown exhibits fourteen irregularly sized tines. 

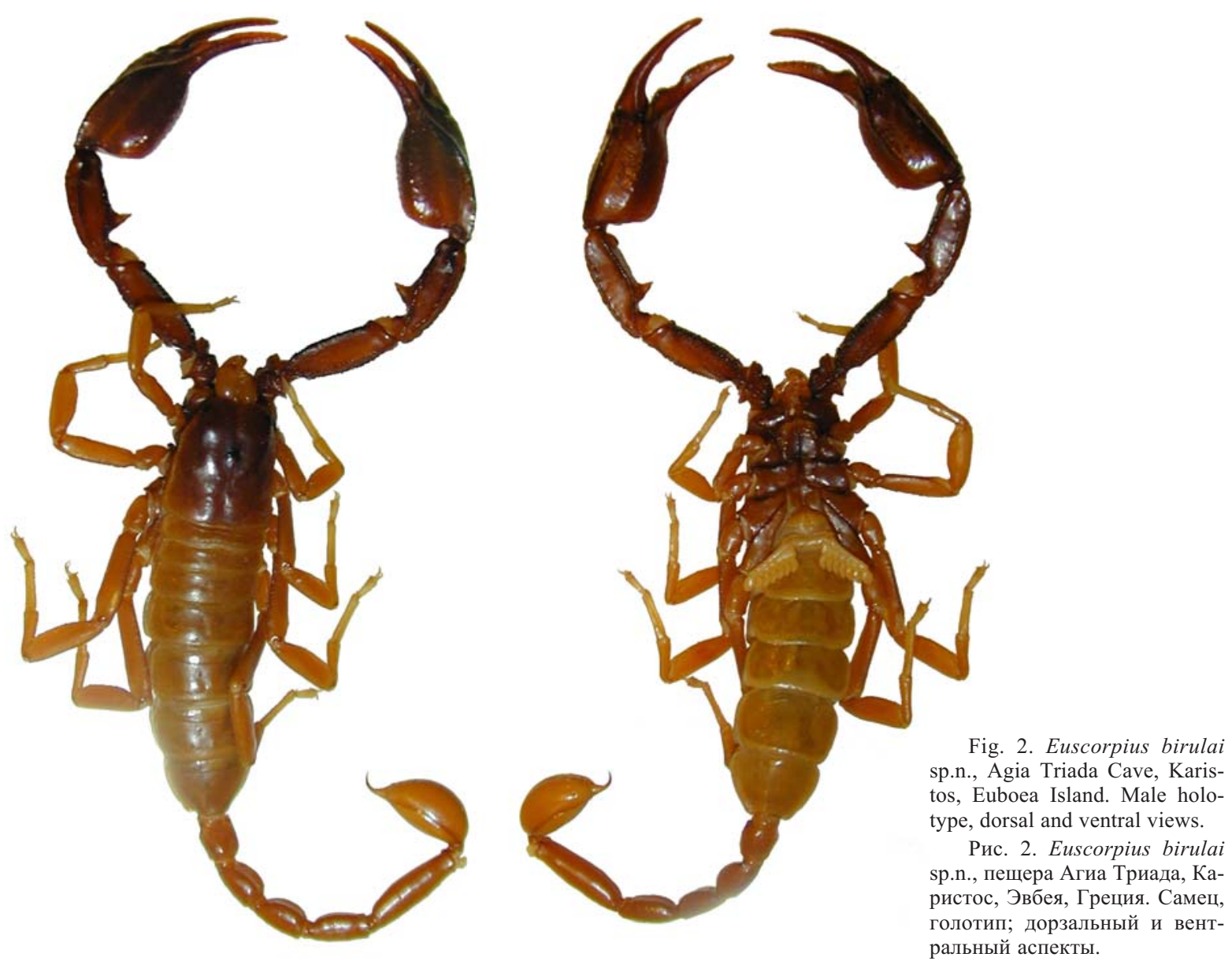

Table 2. Morphometric comparisons between Euscorpius birulai sp.n. and E. koschewnikowi based on dominant morphometrics and their Mean Value Differences (MVD).

Таблица 2. Морфометрическое сравнение Euscorpius birulai sp.n. и E. koschewnikowi, основанное на доминантных морфометрических значениях и их среднем коэффициенте различия (MVD).

\begin{tabular}{|l|c|c|c|}
\hline & $\begin{array}{c}\text { E. birulai }(\mathbf{n}=\mathbf{2}) \mathbf{O}^{7} \\
(\text { mean) }\end{array}$ & $\begin{array}{c}\text { E. koschewnikowi }(\mathbf{n}=1) \\
\text { (mean) }\end{array}$ & MVD \\
\hline Tel_W/Meta1_L & 1.199 & 0.900 & $\Delta 33.2 \%$ \\
\hline Tel_W/Meta2_L & 0.999 & 0.750 & $\Delta 33.2 \%$ \\
\hline Tel_D/ Meta1_L & 1.270 & 0.975 & $\Delta 30.2 \%$ \\
\hline Tel_D/Meta2_L & 0.058 & 0.812 & $\Delta 30.2 \%$ \\
\hline Che_L/ Meta1_L & 5.737 & 4.450 & $\Delta 28.9 \%$ \\
\hline Che_L/Meta2_L & 4.780 & 3.708 & $\Delta 28.9 \%$ \\
\hline MovF_L/ Meta1_L & 3.103 & 2.375 & $\Delta 30.6 \%$ \\
\hline MovF_L/ Meta2_L & 2.582 & 1.979 & $\Delta 30.5 \%$ \\
\hline
\end{tabular}

Tel_W - telson width, Tel_D - telson depth, Che_L — chela length, MovF_L — movable finger length, Meta1_L — metasomal segment I length, Meta2_L - metasomal segment II length.

Tel_W - ширина тельсона, Tel_D - высота тельсона, Che_L — длина клешни, MovF_L — длина подвижного пальца, Meta1_ $\bar{L}$ - длина I сегмента метасомы, Meta2_L — длина II сегмента метасомы. 
Fet et al.

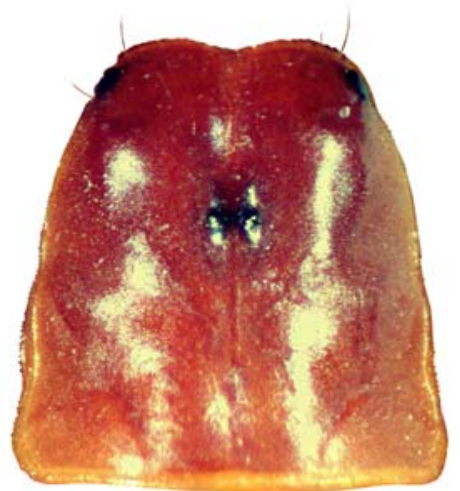

3

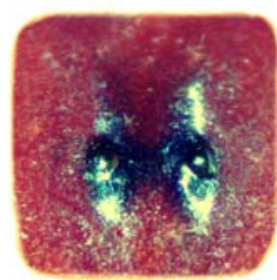

4

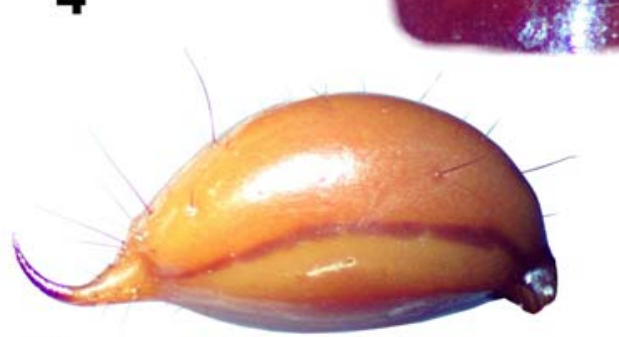

6
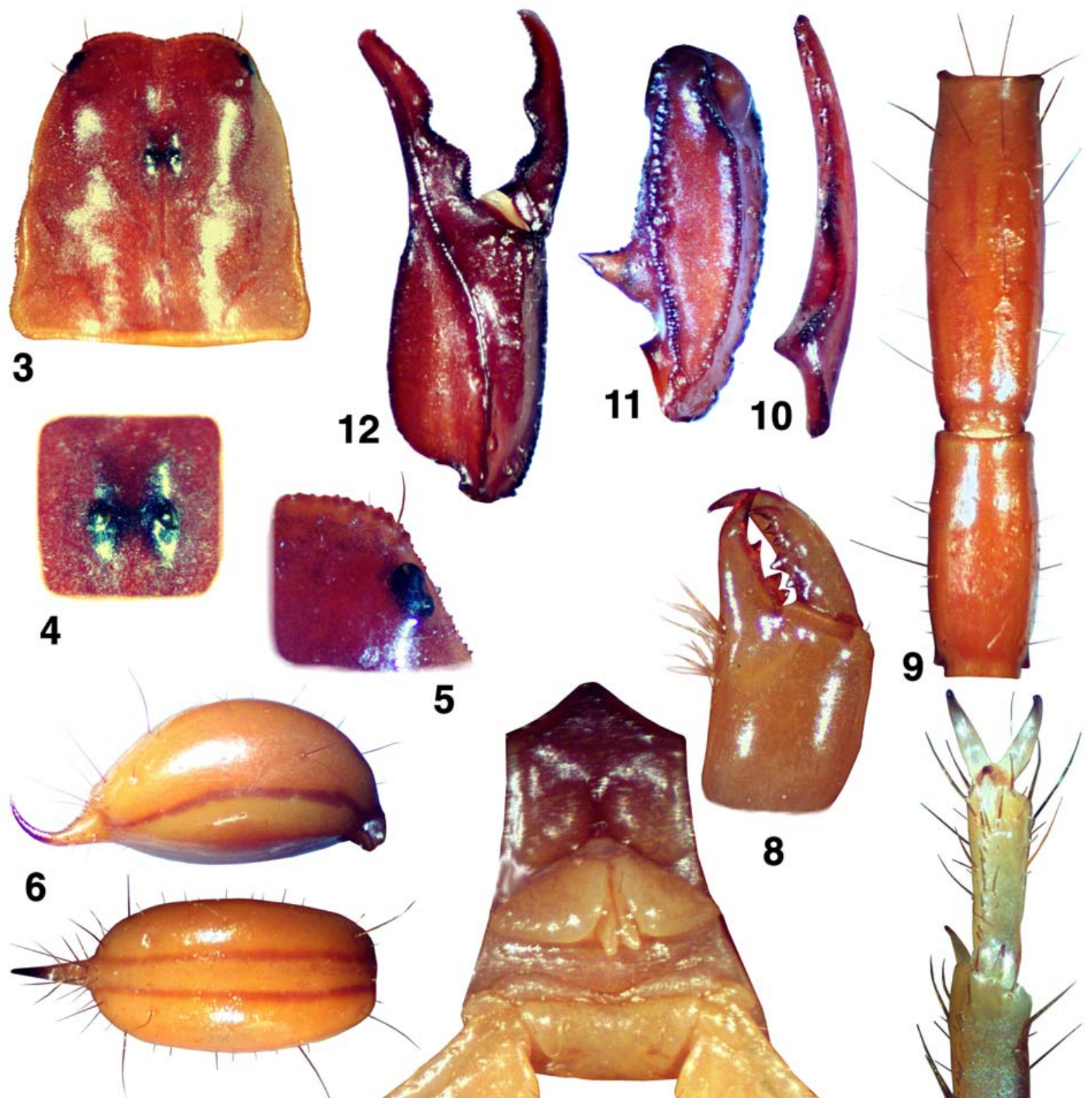

9
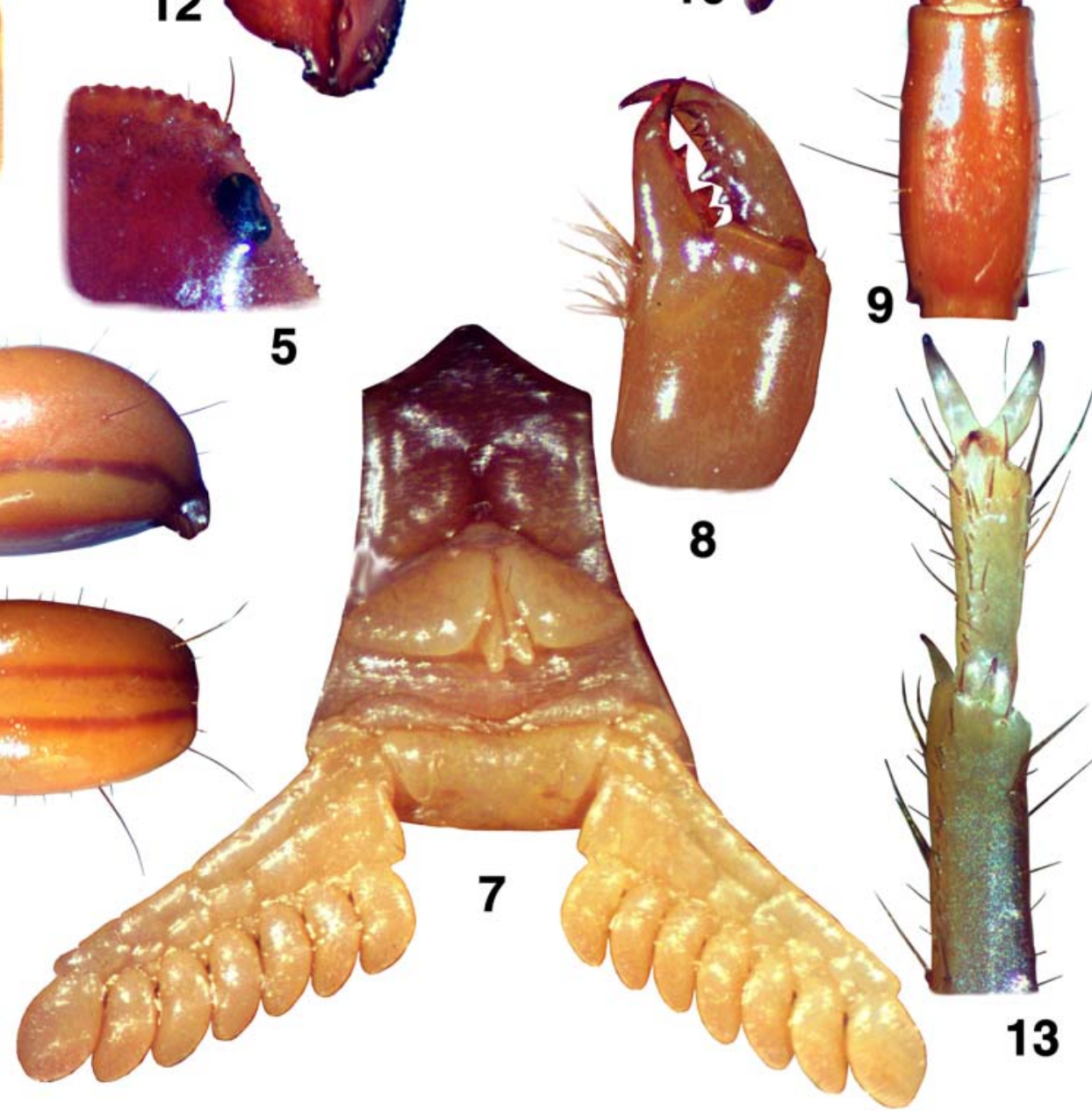

Figs 3-13. Euscorpius birulai sp.n. Agia Triada Cave, Karistos, Euboea Island, Greece, male holotype (3-11), male paratype (12-13): 3 - carapace; 4 - median eyes; 5 - lateral eyes; 6 - telson, lateral and ventral views; 7 - sternopectinal area; 8 - chelicera, dorsal view; 9 - metasomal segments IV-V, ventral view; 10 - movable finger dentition; 11 - patella, dorsal view; 12 - chela, external view; 13 - left leg III tarsus, ventral view.

Рис. 3-13. Euscorpius birulai sp.n., пещера Агиа Триада, Каристос, Эвбея, Греция, самец, голотип (3-11), самец, паратип (1213): 3 - головогрудь; 4 - медиальные глаза; 5 - латеральные глаза; 6 - тельсон, латеральный и вентральный аспекты; 7 стернум и гребневидные органы; 8 - хелицера, дорзальный аспект; 9 - IV-V сегменты метасомы, вентральный аспект; $10-$ зубчики на лезвии подвижного пальца; 11 - пателла, дорзальный аспект; 12 - клешня, внешний аспект; 13 - тарзус ноги III, вентральный аспект. 

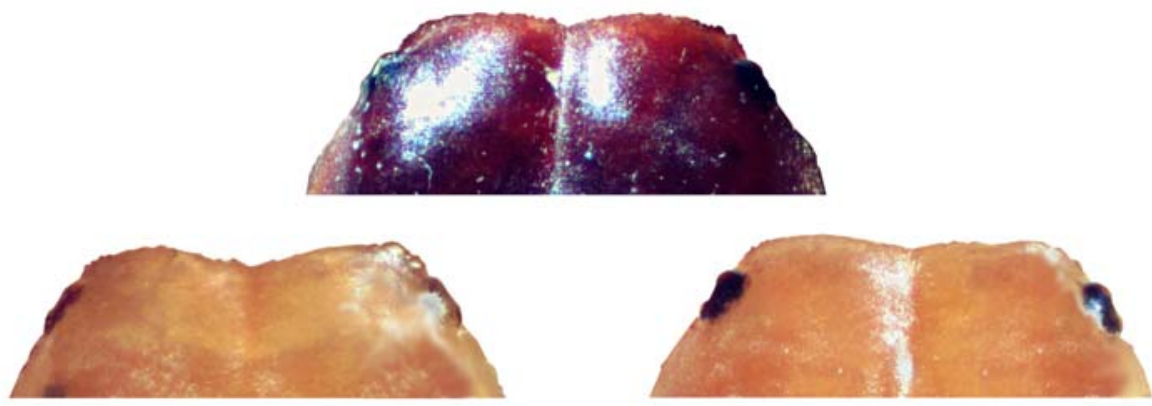

Fig. 14. Euscorpius birulai sp.n., male paratypes, Agia Triada Cave, Karistos, Euboea Island, Greece, showing the anterior median indentation of the carapace. Compare to the male holotype in Fig. 3. Top. Adult. Bottom. Two subadults.

Рис. 14. Euscorpius birulai sp.n., самцы, паратипы, пещера Агиа Триада, Каристос, Эвбея, Греция, Передний медиальный вырез на головогруди. Ср. с самцом (голотип) на Рис. 3. Наверху, взрослый самец; внизу, два субадультных самца.
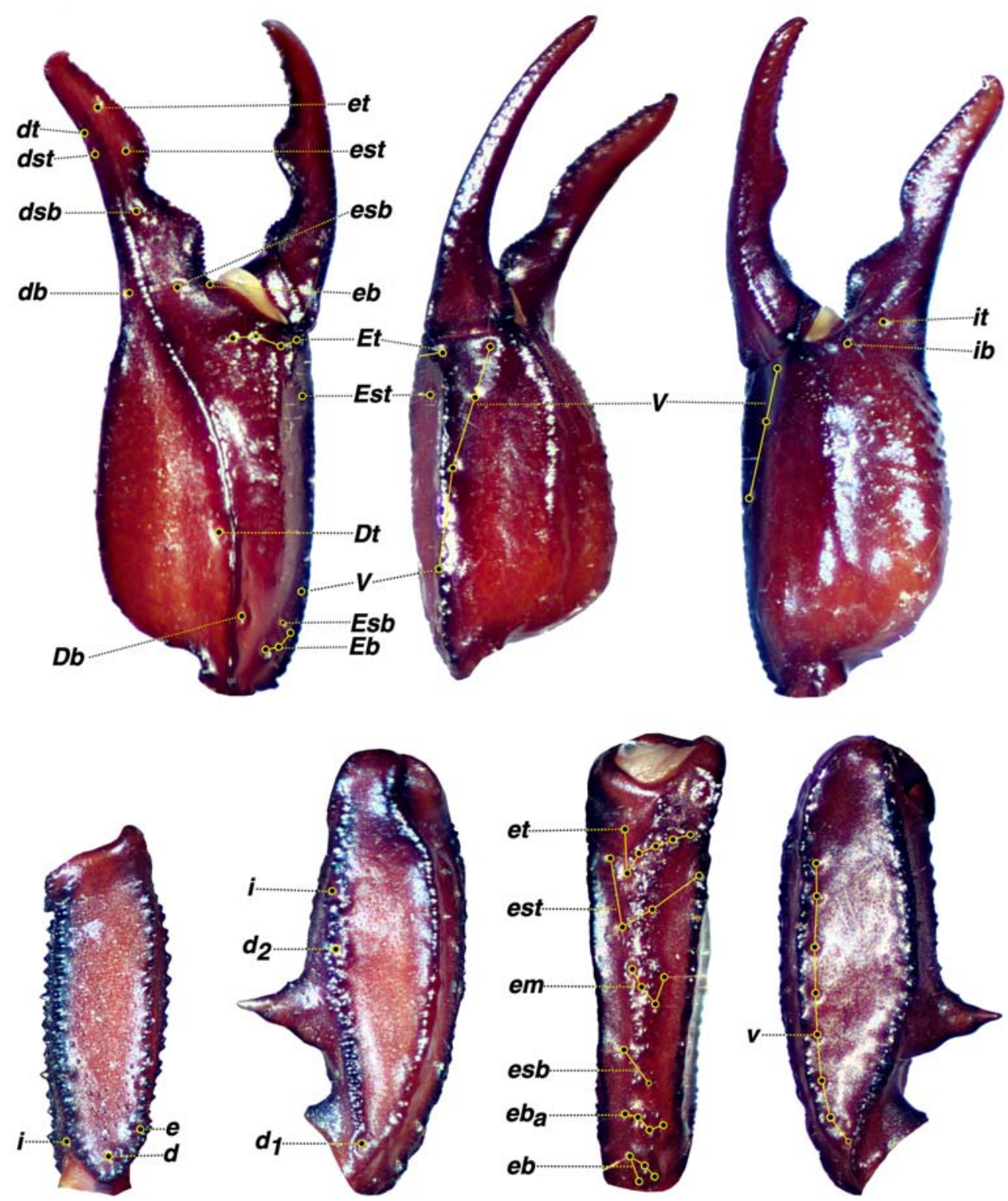

Fig. 15. Euscorpius birulai sp.n., male paratype, Agia Triada Cave, Karistos, Euboea Island, Greece. Trichobothrial pattern.

Рис. 15. Euscorpius birulai sp.n., самец, паратип, пещера Агиа Триада, Каристос, Эвбея, Греция. Расположение трихоботрий. 


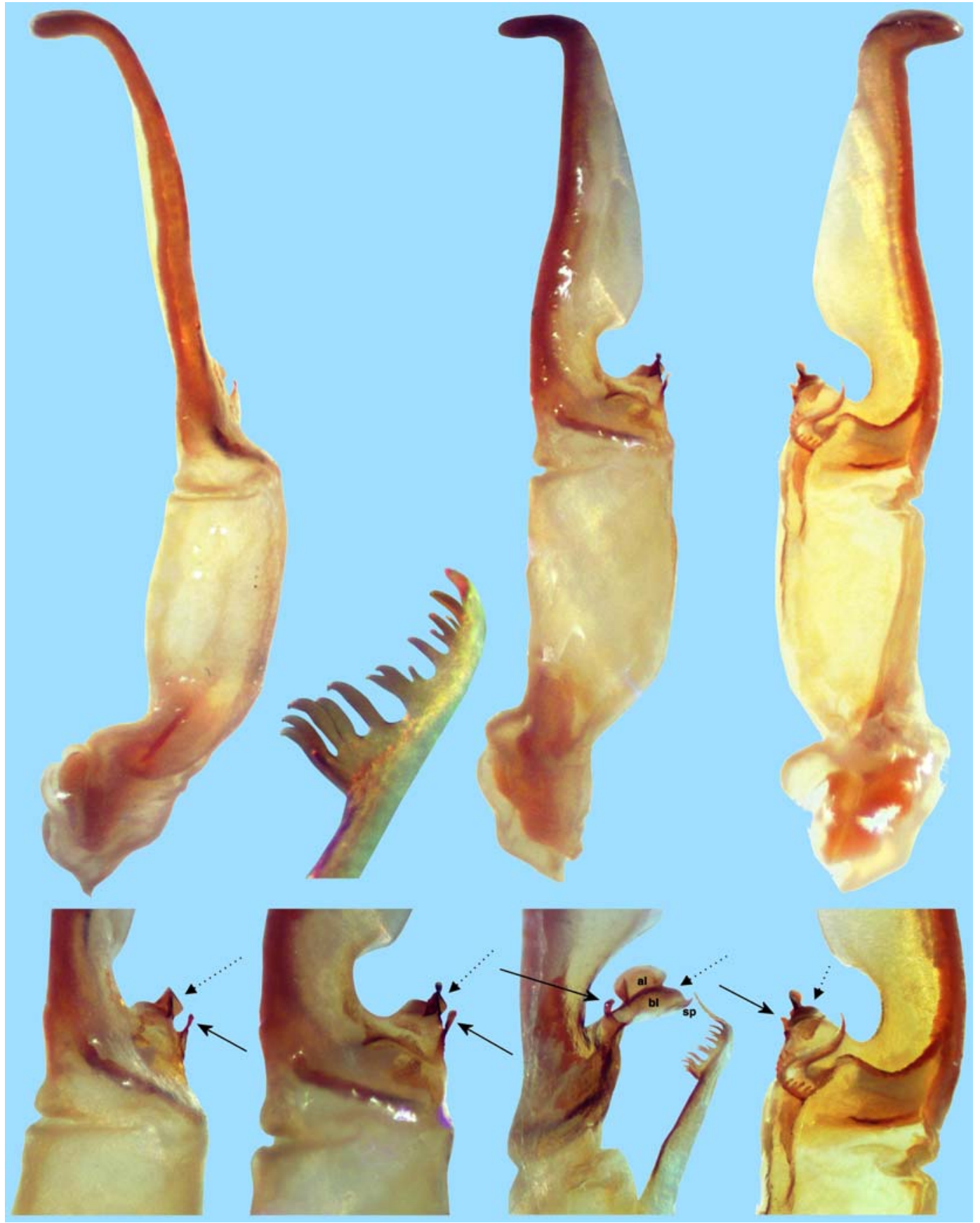

Fig. 16. Euscorpius birulai sp.n., male paratype, Agia Triada Cave, Karistos, Euboea Island, Greece. Right hemispermatophore (photographed submerged in alcohol). Top. Complete structure, external, dorsal, and ventral views. Bottom. Median area close-ups, exterodorsal, dorsal, interodorsal, and ventral views. Dotted arrows indicate primary acuminate process and solid arrows indicate secondary acuminate process. Note, the interodorsal perspective shows the primary acuminate process in full view delineating the basal lobe (bl), auxiliary lobe (al), and spur (sp). Center. Close-up of internal projection crown showing fourteen irregular sized tines.

Рис. 16. Euscorpius birulai sp.n., самец, паратип, пещера Агиа Триада, Каристос, Эвбея, Греция. Правый гемисперматофор (в спирту). Вверху, общий вид: внешний, дорзальный и вентральный аспекты. Внизу, медиальная область, внутренне-дорзальный, дорзальный, внешне-дорзальный вентральный аспекты. Пунктирные стрелки указывают на первичный заостренный отросток; сплошные стрелки указывают на вторичный заостренный отросток. На внутренне-дорзальном аспекте показан полный вид первичного заостренного отростка, включая базальную лопасть (bl), дополнительную лопасть (al) и шпору (sp). В центре, подробный вид внутренней короны, показывающий 14 зубцов различного размера. 


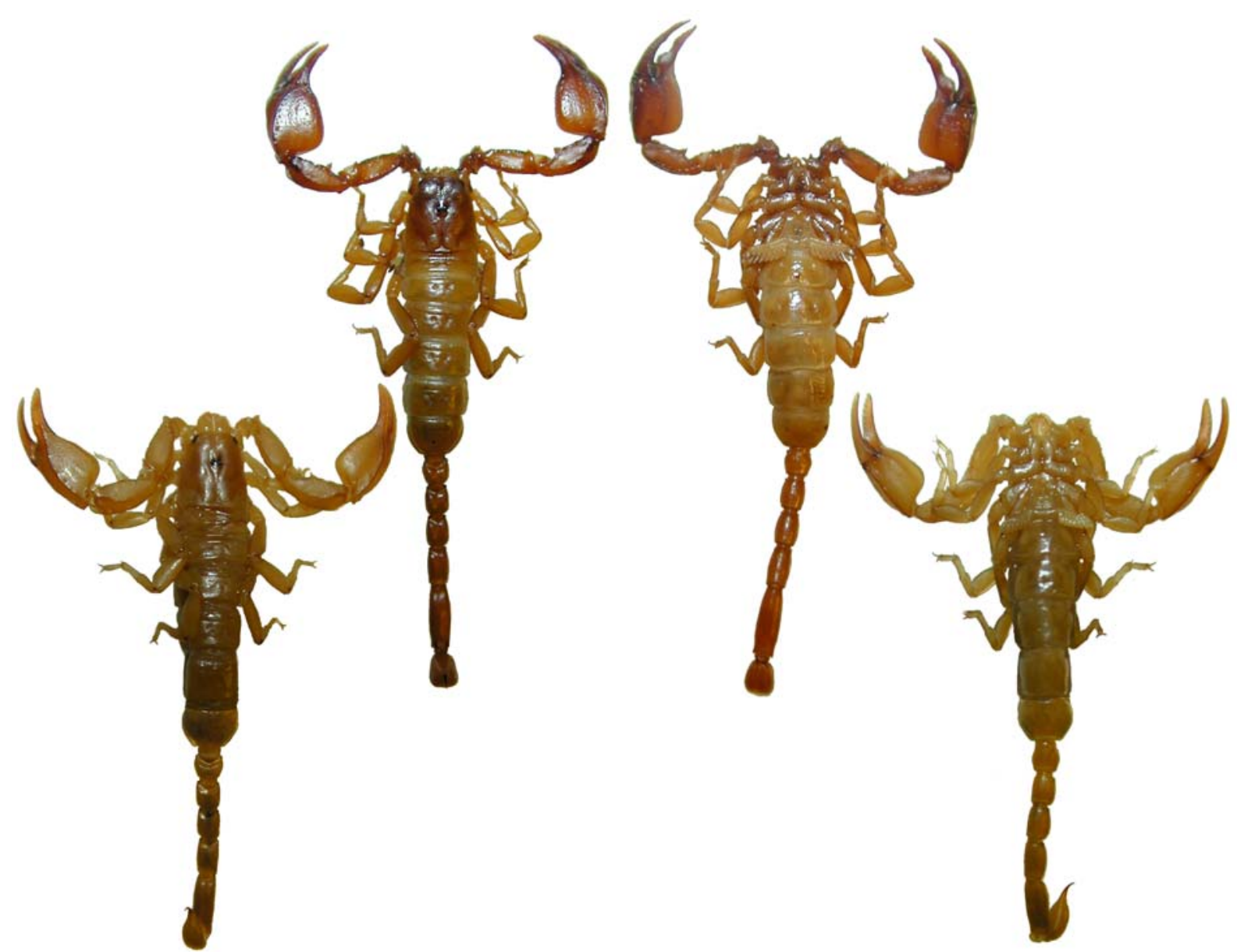

Fig. 17. Euscorpius mylonasi sp.n., Mt. Dirfi, Euboea Island, Greece. Male holotype (top) and female paratype (below), dorsal and ventral views.

Рис. 17. Euscorpius mylonasi sp.n., горы Дирфи, Эвбея, Греция. Самец, голотип (вверху) и самка, паратип (внизу), дорзальный и вентральный аспекты.

Female. Unknown.

Sexual dimorphism. Unknown; only mature males are available.

Variation. We examined eight specimens of E. birulai sp.n. from Euboea ( $\left.5 \sigma^{7} \sigma^{7}, 3 \circ 0\right)$. Variation was as follows: Pectinal teeth number in males, $7[\mathrm{n}=10]$ (a rather low number for the subgenus, known e.g. or $E$. kritscheri and the Italian species E. oglasae Di Caporiacco, 1950); in females, 6 (4) and 7 (2) [n=6], mean 6.33, SD 0.6. Number of ventral patellar trichobothria $(P v)$ was always $8[\mathrm{n}=16]$, Number of external terminal patellar trichobothria (et) was 5 (2) and 6 (14) [n= 16], mean 5.875, SD 0.342 .

\section{COMMENTS}

1. Euscorpius birulai sp.n. is quite similar to $E$. koschewnikowi, a species found on Mt. Athos, Chalkidiki, Central Macedonia, Greece [Fet \& Soleglad, 2002]. Both species are only known from their type locality, roughly $238 \mathrm{~km}$ apart. E. birulai sp.n. was found in a cave, while E. koschewnikowi was apparently not (but see below). Both are dark colored species, the same size (males 32 to $40 \mathrm{~mm}$ ), both are slender, and both have a conspicuously long sharp DPS. Statistically, the pectinal teeth and patellar trichobothrial numbers are in the same range; pectinal teeth $=7-8$ (male), et $=5-$ 6 , and $v=8$. However, E. birulai sp.n. differs from $E$. koschewnikowi with a somewhat wide median indentation on its carapace anterior edge in contrast to an essentially straight edge as seen in the latter (compare Fig. 3 to Fet \& Soleglad [2002: fig. 38]). Morphometrically, we see that $E$. birulai sp.n. has a fatter telson vesicle (width and depth) and a longer chela and movable finger, while E. koschewnikowi has a thinner metasoma, especially the two basal segments, I-II. Based on these observations, we constructed eight morphometric ratios based on these dominant morphometrics and identified the significant differences as shown in Table 2 . We can see that the mean value differences of these eight morphometric ratios ranged from 29 to 33 $\%$, clearly a significant difference. At the same time, it must be noted that this analysis is based only on three sexually mature males, two E. birulai sp.n. and one $E$. 
koschewnikowi, so clearly additional material representing both genders need to be studied.

2. No DNA data are available either for E. birulai sp.n. or for E. koschewnikowi. Phylogenetic position of both these related species is unclear, but judging from their morphology, they most likely do not belong to subgenus Euscorpius s.str. [cf. Tropea, 2013a, 2013b].

3. E. birulai sp.n. is the first Euscorpius species that has been so far found only in a cave (however, directed searches for scorpions have never been conducted in the Mt. Ochi massif outside Agia Triada Cave). It clearly exhibits general attenuation of appendages (pedipalps) although it does not have other typical cave adaptations (depigmentation, eye reduction). Lack of troglobitic forms in Euscorpiidae has been puzzling for the researchers, although some species have been occasionally found in caves [Graham et al., 2012]. Prominent, highly specialized troglomorphic species and genera are known in other chactoid scorpion families (Typhlochactidae, Akravidae) [Fet et al., 2011].

Euscorpius mylonasi Fet, Soleglad, Parmakelis, Kotsakiozi et Stathi, sp.n. Figs 17-31; Table 1. boea).

Euscorpius carpathicus: Kinzelbach, 1975: 32 (in part: Eu-

Euscorpius carpathicus carpathicus: Kritscher, 1993: 384 (in part: Euboea, in part).

Euscorpius carpathicus: Stathi \& Mylonas, 2001: 289 (in part: Euboea; Stouronisi islets).

Euscorpius sp. clade E3: Parmakelis et al., 2013: 10 (in part: Euboea).

TYPE MATERIAL: Holotype Or, GREECE, Central Greece: Euboea (=Evvoia) Island, Mt. Dirfi, road Steni Dirfios-Agia Irini, $1080 \mathrm{~m}$, W slope, 6 June 1999-17 June 2000, leg. P. M. Giachino \& D. Vailati (MRSN, No. 14). Paratypes (16 $\mathrm{O}^{7} \mathrm{O}^{7}, 15$ 우, $1 \mathrm{im}$.) same label as holotype, 3 오 (MRSN, No. 14), $3 \mathrm{O}^{7} \mathrm{O}^{7}$ (MRSN, No 12), 1 O 2 우 (MRSN, No. 10); Mt. Dirfi, road Steni Dirfios-Agia Irini, $1080 \mathrm{~m}$, W slope, 9 June 2002, leg. P.M. Giachino \& D Vailati, $2 O^{x} \sigma^{x} 1$ ( 1 CSNG, PMGC Sc60); Mt. Dirfi, road Steni Dirfios-Agia Irini, $1000 \mathrm{~m}$ a.s.l., ENE slope, 9 June 2002, leg. P.M. Giachino \& D. Vailati, $10^{7}$ (MCSNG, PMGC Sc58); Mt. Dirfi, road Steni Dirfios-Agia Irini, $1100 \mathrm{~m}$, ENE slope, $38^{\circ}$ $37^{\prime} 09.51^{\prime \prime} \mathrm{N} 23^{\circ} 51^{\prime} 12.70^{\prime \prime} \mathrm{E}, 10$ June $2002-17$ June 2004, leg. P.M. Giachino \& D. Vailati, 2 o $^{7} 1$ \& sbad (MCSNG, PMGC Sc59); Mt. Dirfi, under Steni Dirfios, 830 m, 28 May 1999, leg. P.M Giachino, 1 o juv. (MRSN); Mt. Xerovouni, N slope, $1100 \mathrm{~m}$, $38^{\circ} 35^{\prime} 17.46^{\prime \prime} \mathrm{N} 23^{\circ} 54^{\prime} 27.03^{\prime \prime} \mathrm{E}, 8$ June 2002, leg. P.M. Giachino \& D. Vailati, 1 O $^{7} 1$ क (MCSNG, PMGC Sc62); Mt. Dirfis (=Mt Dirfi), 1100 m, 16 May 1989, leg. E. Kritscher, $10^{7}$ sbad (NHMW 15983); Pano Steni, 25 April 1978, leg. M. Mylonas, 2 우 im. (NHMC); Stouronisi islets, south of Euboea, 4 May 1991, 1 + (NHMC); Valaha islet, 21 January 2002, leg. K. Triantis, 1 juv. (NHMC 3306); Nea Styra, 14 April 2006, leg. K. Vardinoyannis, 1 o (NHMC 7320, used for DNA as S2); Agios Andreas islet (between mainland and Euboea), $38^{\circ} 10^{\prime} \mathrm{N}, 24^{\circ} 09^{\prime} \mathrm{E}, 5 \mathrm{~m}, 15$ April 2006, leg. K. Vardinoyannis, $4 \mathrm{O}^{7} \mathrm{O}^{7} 1$ क (NHMC 7323, 81.1.1.69, Eus86; used for DNA as S1); Euboea, south, no date/collector, 1 + (ZMHB 15275).

DNA SEQUENCES [Parmakelis et al., 2013]: GREECE, Central Greece: Euboea (=Evvoia) Island, Nea Styra, 14 April 2006 , leg. K. Vardinoyannis, 1 क (NHMC 7320, S2), geneseq-2 16S, COI, COII, ITS1 (GenBank accession numbers KC215657, KC215743, KC215828, KC215913); Agios Andreas islet, 15 April 2006, leg. K. Vardinoyannis, 1 O (NHMC 7323, Eus86, S1): geneseq-2 16S, COI, COII, ITS1 (GenBank accession numbers KC215658, KC215744, KC215829, KC215914).

DIAGNOSIS. A brown (mesosoma and legs lighter), without contrasting patterns, small species (28-31 $\mathrm{mm}$ for adult males). Pectinal tooth count usually 8 in males, 7-8 in females (mean 7.33). Anterior edge of carapace straight. Patellar trichobothria number: et usually 5 and $v=7-8$ (mean 7.39); est - est $_{4}$ angled at $e s t_{3}$. Dorsal patellar spur (DPS) moderately developed. Metasomal segments I-IV with dorsal carinae rounded and smooth, ventrolateral carinae vestigial and smooth; segment $\mathrm{V}$ with dorsolateral carinae slightly granulated distally, ventrolateral carinae slightly granulated; lateral and ventromedian carinae absent on all segments.

ETYMOLOGY. The species name is a patronym honoring Dr. Moysis Mylonas (University of Crete, Heraklion, Crete, Greece), a prominent Greek zoologist whose tireless field work and collections over many decades greatly contributed to our knowledge of Aegean scorpions.

DISTRIBUTION. Known only from Euboea Island (and nearby islets) in the western part of the Aegean Sea (see map in Fig. 1).

DESCRIPTION. The following description is based primarily on the holotype male from Mt. Dirfi, Euboea Island, Greece, with additional information derived from paratype male and female. Measurements of the holotype, and paratype male and female are presented in Table 1. See Fig. 17 for dorsal and ventral views of the male holotype and female paratype.

Coloration. Carapace, pedipalps, metasoma, and telson brown; tergites, legs, sternites, and chelicerae light brown; leg coxae and sternum brown; genital operculum, pectines, basal piece yellow-brown. No patterns present.

Carapace (Fig. 18). Anterior edge straight; interocular area smooth and lustrous, lacking any indication of carinae; median to posterior area rough in appearance. Ttwo lateral eyes. Median eyes and tubercle are small in size, positioned anterior of middle with the following length and width ratios: 0.424 (anterior edge to medium tubercle middle / carapace length) and 0.157 (width of median tubercle including eyes / width of carapace at that point).

Mesosoma. Tergites I-VII essentially smooth; tergite VII lacking lateral and median carinal pairs. Sternites III-VII smooth and lustrous; VII lacking lateral and median carinae. Stigmata very small, narrow elliptical.

Metasoma (Fig. 22). Segment I wider than long in ratio 0.875 . Segments I-IV: dorsal carinae rounded and smooth; dorsolateral and lateral carinae obsolete; ventrolateral carinae vestigially present and smooth; single ventromedian carina obsolete. Segment V: dorsolateral carina slightly granulated distally; lateral carinae obsolete; ventrolateral slightly granulated; and ventromedian carinae obsolete. Anal arch with approximately 14 small pigmented granules. Intercarinal areas smooth.

Telson (Fig. 23, paratype female Fig. 24). Vesicle swollen and elongated, with short highly curved ac- 


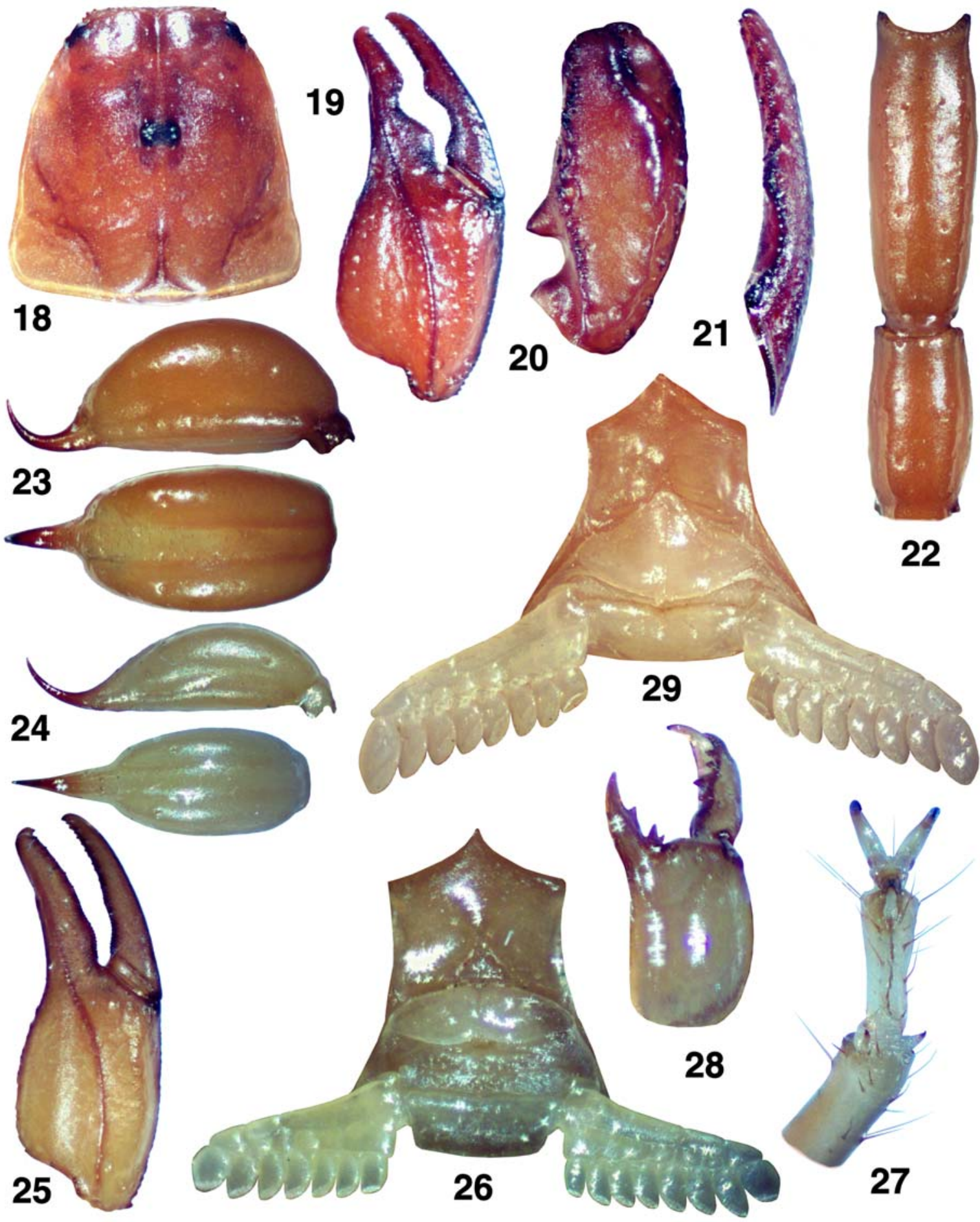

Figs 18-29. Euscorpius mylonasi sp.n. Mt. Dirfi, Euboea Island, Greece, male holotype (18-23), female paratype (24-26), male paratype (27-28); Agios Andreas islet, Euboea Island, Greece, male paratype (29). 18 - carapace; 19 - chela, external view; 20 patella, dorsal view; 21 - movable finger dentition; 22 - metasomal segments IV-V, ventral view; 23 - telson, lateral and ventral views; 24 - telson, lateral and ventral views; 25 - chela, external view; 26 - sternopectinal area; 27 - right leg IV tarsus, ventral view; 28 chelicera, dorsal view; 29 - sternopectinal area.

Рис. 18-29. Euscorpius mylonasi sp.n., горы Дирфи, Эвбея, Греция, самец, голотип (18-23), самка, паратип (24-26), самец, паратип (27-28); островок Агиос Андреас, о-в. Эвбея, Греция, самец, паратип (29). 18 — головогрудь; 19 - клешня, внешний аспект; 20 - пателла, дорзальный аспект; 21 - зубчики на лезвии подвижного пальца; 22 - IV-V сегменты метасомы, вентральный аспект; 23 - тельсон, латеральный и вентральный аспекты; 24 - тельсон, латеральный и вентральный аспекты; $25-$ клешня, внешний аспект; 26 - стернум и гребневидные органы; 27 - тарзус правой ноги III, вентральный аспект; 28 хелицера, дорзальный аспект; 29 - стернум и гребневидные органы. 

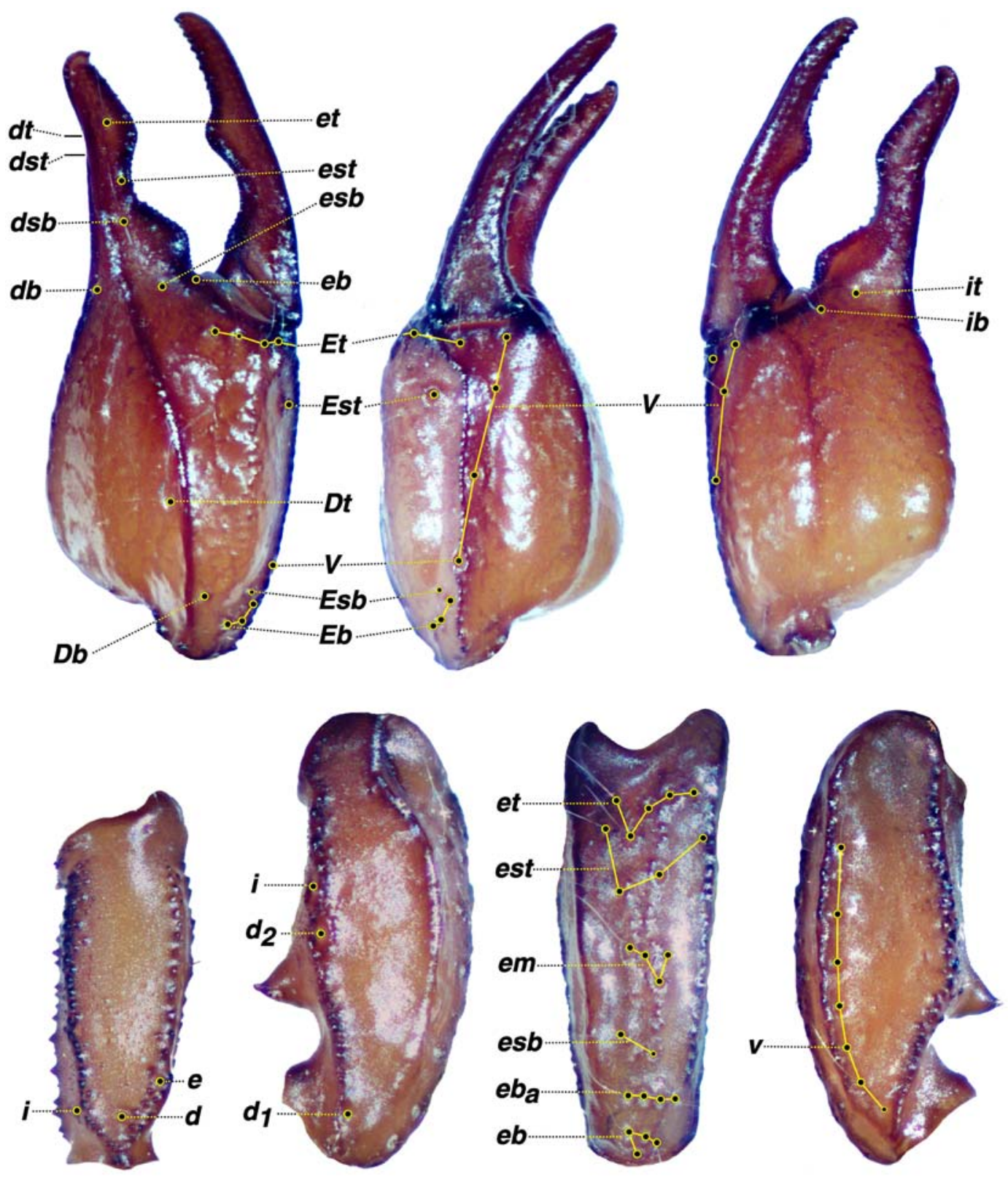

Fig. 30. Euscorpius mylonasi sp.n., male paratype, Mt. Dirfi, Euboea Island, Greece. Trichobothrial pattern.

Рис. 30. Euscorpius mylonasi sp.n., самец, паратип, горы Дирфи, Эвбея, Греция. Расположение трихоботрий.

uleus. Vesicle essentially void of granules, very lustrous. Vesicular tabs vestigial and smooth.

Pectines (Fig. 29, paratype male; paratype female Fig. 26). Medium-developed segments exhibiting length / width ratio 2.206 (length taken at anterior lamellae / width at widest point including teeth). Sclerite construction complex, three anterior lamellae and 5/6 middle lamella; fulcra of medium development. Teeth number $8 / 8$. Sensory areas developed along distal aspect on all teeth, including basal tooth. Basal piece large, with subtle shallow indentation along anterior edge, length / width ratio 0.371 .

Chelicerae (Fig. 28, male paratype). Movable finger dorsal edge with two small subdistal $(s d)$ denticles; ventral edge smooth; serrula not visible. Ventral distal denticle $(v d)$ conspicuously longer than dorsal $(d d)$. Fixed finger with four denticles, median $(\mathrm{m})$ and basal (b) denticles conjoined on common trunk; no ventral accessory denticles present.

Pedipalps (Figs 19-21, paratype female Fig. 25). Well-developed chelae, moderately carinated, strong scalloping on chelal fingers. Femur: dorsointernal and ventrointernal carinae heavily serrated, dorsoexternal irregularly serrated, and ventroexternal rounded and smooth. Dorsal surface covered with small granules proximally, ventral surface scattered with small granules on proximal half, internal and external surfaces 


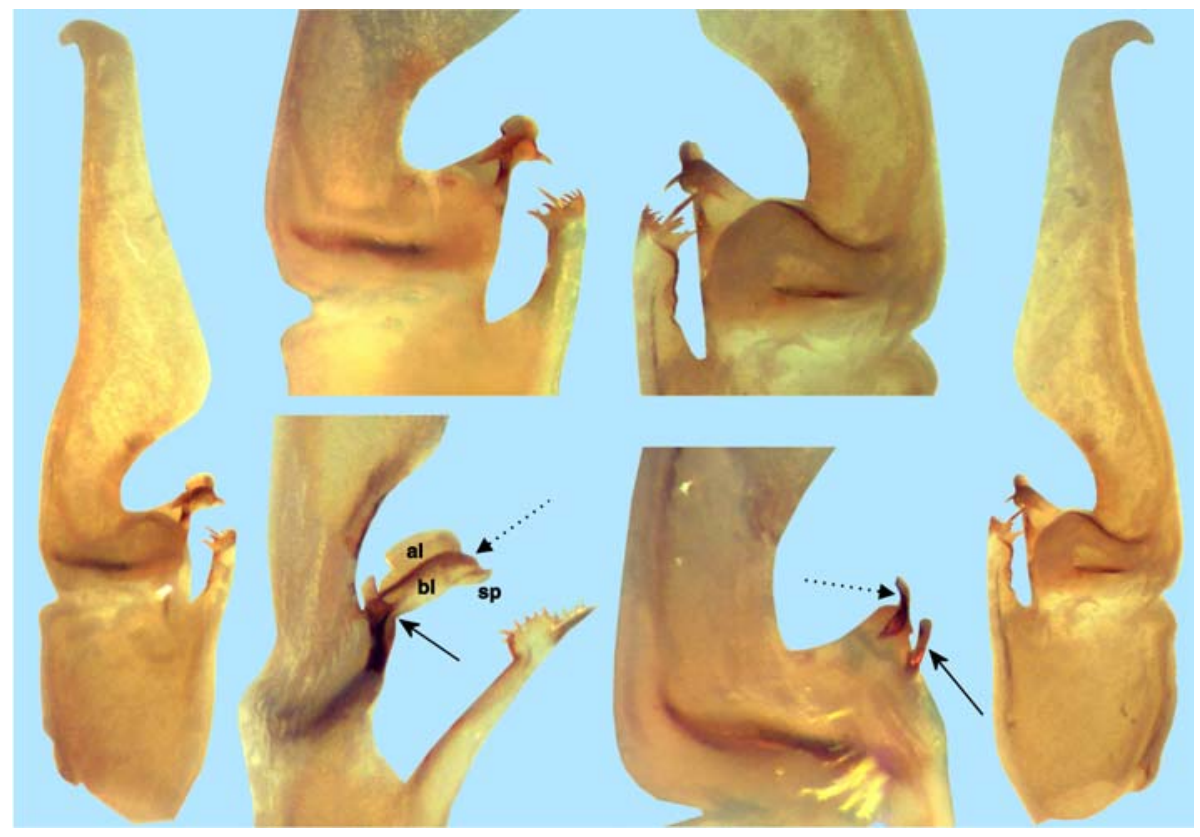

Fig. 31. Euscorpius mylonasi sp.n., male paratype, Mt. Dirfi, Euboea Island, Greece. Right hemispermatophore (photographed submerged in alcohol). Left. Dorsal view. Right. Ventral view. Top. Close-up of median area, dorsal and ventral views. Bottom. Close-up of median area, interodorsal and exterodorsal views, showing different perspectives of the two acuminate processes. Dotted arrows indicate primary acuminate process and solid arrows indicate secondary acuminate process. Note, the interodorsal perspective shows the primary acuminate process in full view delineating the basal lobe (bl), auxiliary lobe (al), and spur (sp).

Рис. 31. Euscorpius mylonasi sp.n., самец, паратип; горы Дирфи, Эвбея, Греция. Правый гемисперматофор (в спирту). Слева: дорзальный аспект. Справа: вентральный аспект. Вверху: медиальная область, внутренне-дорзальный и внешне-дорзальный аспекты; два заостренных отростка показаны под разными углами. Пунктирные стрелки указывают на первичный заостренный отросток; сплошные стрелки указывают на вторичный заостренный отросток. Полный вид первичного заостренного отростка во внутренне-дорзальном аспекте показывает базальную лопасть (bl), дополнительную лопасть (al) и шпору (sp).

each with a row of serrated granules. Patella: dorsointernal and ventrointernal carinae strongly crenulated, dorsoexternal rough but not granulate, ventroexternal generally smooth with some scattered granules, and exteromedian carina not overly developed with some scattered granules. Dorsal surface rough but with no granules and ventral smooth; external rough with irregular granulation; internal surface smooth with moderately developed DPS and near obsolete VPS (single small granule). Chelal carinae: Complies with the "10carinae configuration". Digital (D1) carina strong, lowprofile polished granulation; subdigital (D2) essentially obsolete, represented by two granules; dorsosecondary (D3) near obsolete, area quite flat, presented by two small basal granules; dorsomarginal (D4) strong, rounded, and irregularly granulated; dorsointernal (D5) obsolete to highly rounded, covered with granules; ventroexternal (VI) strong, evenly granulated, curving to external condyle of movable finger; ventromedian $(V 2)$ obsolete; ventrointernal (V3) rounded with scattered granulation on distal half; external $(E)$ irregularly developed with rough surface. Chelal finger dentition: Median denticle $(M D)$ row groups in straight line; 6/6 $I D$ s fixed finger and $6 / 7$ on movable finger; $5 / 5 O D$ s on fixed finger and 7/7 on movable finger; $4 / 4$ and $5 / 5$ IADs on fixed and movable fingers, respectively. Trichobothrial patterns (Fig. 30, paratype male): Type C, neobothriotaxic: chela ventral $=4 / 4$; patellar $e b=4 / 4$, $e b_{a}=4 / 4, e s b=2 / 2, e m=4 / 4$, est $=4 / 4$, et $=5 / 5$; patellar ventral $=7 / 7$; est $_{2}$-est $t_{4}$ angled at est $_{3}$.

Legs (Fig. 27, male paratype). Both pedal spurs present on all legs, lacking spinelets; tibial spurs absent. Tarsus with single row of spinules on ventral surface, terminating distally with a well-developed spinule pair. Unguicular spine well-developed and pointed.

Hemispermatophore (FIG. 31, male paratype). Hemispermatophore with a well-developed truncal flexure. Lamina with a conspicuous basal constriction, terminus tapered and curving towards the exteroventral surface. Median projection with both primary and secondary acuminate processes. The basal and auxiliary lobes and spur are present on the primary acuminate process. The internal projection crown exhibits approximately eleven irregularly sized tines.

SEXUAL DIMORPHISM. The adult female exhibits a weak subtle proximal gap and movable finger lobe on the chela, whereas they are well-developed in the male; the genital operculum sclerites in the female are connected along the middle, not separated as in the male; genital papillae are absent in the female, present in the male. The pectinal tooth counts are lower in females, 6-8 (7.33) [12] as compared to 7-9 (8.00) [12] in males, a $9.14 \%$ difference in the means. The telson vesicle in the female is not as swollen as it is in the male; the telson length compared to its depth is 3.263 in the female and 2.469 in the male, exhibiting a 
$32.2 \%$ difference. The chelal palm in the female is not as swollen as it is in the male, the chelal length compared to its depth is 2.644 in the female, and 2.394 in the male, a $10.4 \%$ difference, respectively.

VARIATION. We examined 12 specimens of $E$. mylonasi sp.n. from Euboea (6 $\sigma^{7} \sigma^{7}, 6$ + 9 ). Variation was as follows: pectinàl teeth number in males, 7 (2), 8 (8), 9 (2) [n=12], mean 8.00, SD 0.60; in females, 6 (1), 7 (6), 8 (5) [n=12], mean 7.33, SD 0.65. Number of ventral patellar trichobothria $(P v)$ was $7(16), 8(5)$ and 9 (2) $[\mathrm{n}=23]$, mean 7.39, SD 0.66. Number of external terminal patellar trichobothria (et) was 4 (2), 5 (17), 6 (4) $[\mathrm{n}=23]$, mean 5.09, SD 0.51.

\section{COMMENTS}

1. Several methods of species delimitation and a species validation method were employed in Parmakelis et al. [2013] based on the phylogeny inferred using sequence data from one nuclear and three mtDNA loci. In all the species delimitation methods, the Euscorpius mylonasi sp.n. described herein, was always strongly supported as corresponding to an independent evolutionary unit. In the resulting phylogeny [Parmakelis et al., 2013], E. mylonasi sp.n. described in this work was not placed within the nominotypic subgenus Euscorpius s.str. but formed part of a basal clade E3 along with Skyros Island population (see below).

2. On Euboea, E. mylonasi sp.n. is also sympatric with a Greek form of a widespread "Euscorpius sicanus complex", easily distinguishable by trichobothrial series $e b=5$ (see Parmakelis et al., 2013). We identified the latter form among NHMW specimens collected by E. Kritscher in the localities Mirtias (1 $\sigma^{7}, 20$ May 1989, NHMW 15984) and Voutas (1 B\&, 23 May 1989 , NHMW 15985). Both were reported under " $E$. carpathicus carpathicus" [Kritscher, 1993]. We also identified a previously unpublished specimen belonging to "E. sicanus complex" from an islet off southern Euboea (Karistos, Mikro Kouneli islet, 13 May 2005, leg. K. Vardinoyannis, NHMC 7947); this form seems to have colonized many small islands across the Aegean Sea [Parmakelis et al., 2013].

\section{Euscorpius cf. mylonasi}

Euscorpius sp. clade E3: Parmakelis et al., 2013: 10 (in part: Skyros).

MATERIAL. GREECE, Central Greece, Skyros Island, all leg. by K. Triantis: Achili maquis, $43 \mathrm{~m}$, 20 January 2002, 1 q (NHMC 3301, Eus13); Acherounes, $500 \mathrm{~m}$ before the beach, 20 January 2002, 2 우우 (NHMC 3304, Eus14-15); Peramata to Koumari pine forest, $52 \mathrm{~m}, 22$ January 2002, 1 + (NHMC 3310, Eus16); Priona District, $2 \mathrm{~km}$ before Agios Efstratios, $160 \mathrm{~m}, 24$ January 2002, 2 우 (NHMC 3319, Eus17-18); Mesa Diavatis islet, 10 May 2002, 1 $0^{7}$ sbad, 1 + (NHMC 3231, Eus11-12); Mt. Kochilas, 792 m, 13 May 2002, 3 우 (NHMC 3242, Eus8-10).

DNA SEQUENCES [Parmakelis et al. 2013]. GREECE, Central Greece, Skyros Island, Peramata to Koumari pine forest, $52 \mathrm{~m}$, 22 January 2002, 1 + (NHMC 3310, Eus16, E07); geneseq-3 16S, COI, COII, ITS1 (GenBank accession numbers KC215593, KC215676, KC215762, KC215847).

COMMENTS. No information about Euscorpius from Skyros exists in literature. All studied specimens from Skyros were collected by K. Triantis in 2002 as a part of a detailed ecological survey of this island; for a detailed discussion see Triantis et al. [2005]. The Skyros population forms a sister group to $E$. mylonasi sp.n. from Euboea within a well-defined DNA clade E3 [Parmakelis et al., 2013]; the two exhibit considerable genetic divergence. Also, meristic traits differed considerably in these two populations, in particular number of ventral patellar trichobothria (mean 8.05 on Skyros versus 7.39 of E. mylonasi sp.n.) and external patellar trichobothria (mean 6.14 on Skyros versus 5.09 of E. mylonasi sp.n). Skyros population could potentially represent a separate species; however, its status at this moment is unclear due to the lack of adult males for morphological analysis.

VARIATION. We examined 11 specimens from Skyros (1 $\bigcirc^{7}, 10$ 우). Variation was as follows: Pectinal teeth number in one available subadult male was 9 (1), 10 (1) $[\mathrm{n}=2]$; in females, 6 (2), 7 (17), 8 (1) $[\mathrm{n}=$ 20], mean 6.95 , SD 0.39. Number of ventral patellar trichobothria $(P v)$ was $7(2), 8(16)$ and $9(3)$ [n = 21], mean 8.05 , SD 0.50 . Number of external terminal patellar trichobothria (et) was 6 (18) and 7 (3) [n=21], mean 6.14, SD 0.36.

\section{Biogeography}

The island fauna of Euscorpius in Greece is very poorly explored. Only a few of Euscorpius species are currently known to be island endemics: of Crete (E. candiota), Corfu (E. corcyraeus), and Tinos (E. kritscheri); an undescribed endemic population ( $E$. cf. kritscheri) is also found on Andros [Fet et al., 2013a]. Other taxa found on islands are also present on the mainland: such are E. naupliensis and E. hadzii (both found on Zakynthos), E. scaber (Thasos), E. avcii (Samos), "E. tauricus complex" (Cyclades), and "E. sicanus complex" (many islands from the Sporades to Kalymnos) [Fet et al., 2013a; Parmakelis et al., 2013].

As many other Greek islands [Triantis \& Mylonas, 2009], Euboea is a well-known center of endemism, with 39 endemic species of plants belonging to 23 genera and 14 families but no endemic genera [Trigas \& Iatrou, 2006]. Among animals, prominent endemic forms are known such as beetles (Henrotiella eubeensis, Cholevidae) [Perreau, 1999], land snails (Mastus dirphicus, Enidae) [Parmakelis et al., 2005], and cicadas (Cicadidae), including an endemic genus Euboeana [Gogala et al., 2011].

Cave fauna of Euboea also has attracted considerable attention. From Agia Triada Cave, an endemic cave cricket Dolichopoda cassagnaui Boudou-Saltet, 1973 has been known [Rampini et al., 2012]. An endemic mollusk genus Speleodentorcula beroni (now in Argnidae) [Gittenberger, 1985] from Skotini Cave on Euboea was collected by Petar Beron (Sofia, Bulgaria). Beron's great scorpion collection also provided us with the holotype and paratypes of Euscorpius birulai sp.n. found in 2003 from Agia Triada Cave. Independently, this cave's fauna was explored in 2002 by Pier 
Mauro Giachino and Dante Vailati (Torino, Italy). Their collections yielded additional paratypes of Euscorpius birulai sp.n., as well as a remarkable new cave genus and species of carabid beetles, Hystricosphodrus vailatii [Casale \& Giachino, 2004]. The latter authors noted the high zoogeographic interest of their endemic cave taxon "as extant relict of a primitive colonization by Aegean elements, survived in subterranean environment and in insular condition, presently sympatric in the Evvoia (Evia) Island with elements of more recent origin and closely related to continental taxa." The same situation can be seen in the genus Euscorpius, where a non-cave species E. mylonasi sp.n. is more widely found across the island, while an apparent relict E. birulai sp.n. so far is limited to Agia Triada Cave in the south. According to P. M. Giachino (pers. comm.), the scorpions were found at about $10-15 \mathrm{~m}$ of depth in one of the inactive, lateral (dry) galleries (the Agia Triada has a very active spring with about $1 \mathrm{~m}^{3} / \mathrm{min}$ of water flow). The associated fauna was represented by cave endemics Hystricosphodrus vailatii and Henrotiella eubeensis. P. M. Giachino also suggests that Mt. Ochi massif, where the Agia Triada Cave is located, has a completely different (and probably more ancient) fauna from that of Mt. Dirfi.

A very intriguing fact is the morphological similarity between $E$. birulai sp.n. and poorly known $E$. koschewnikowi. The latter is known only from a very isolated portion of Northwestern Greece - Mount Athos ("Monastic State of Agion Oros"), an autonomous state (polity) under Greek jurisdiction, located on the easternmost "leg" of Chalkidiki Peninsula. While apparently a non-cave species, E. koschewnikowi could occupy a secluded habitat; it was never collected since its original description [Fet \& Soleglad, 2002], even by one of the best Euscorpius collectors, Erich Kritscher. He visited Mount Athos in July 1976 [Kritscher, 1993] but collected only E. scaber Birula, 1900, which is a quite different, non-cave species [Fet et al., 2013b].

ACKNOWLEDGEMENTS. We are grateful to many colleagues who kindly loaned and shared types and comparative material with us, and helped in field collection and lab work, including Petar Beron, Alberto Chiarli, Christo Deltshev, Giuliano Doria, Jason Dunlop, Pier Mauro Giachino, Jürgen Gruber, Christoph Hörweg, Dimitris Kaltsas, Moysis Mylonas, Roberto Poggi, Alexi Popov, Stavroula Poulikarakou, Verena Stagl, Pavel Stoev, Maria Tavano, Kostas Triantis, Dante Vailati, and Katerina Vardinoyannis. Field work of P.M. Giachino and D. Vailati in Greece was part of the program "Research Missions in the Mediterranean Basin" sponsored by the World Biodiversity Association ONLUS, XXXII contribution. Special thanks are to Fulbright Scholar Program (Council for International Exchange of Scholars, USA), Fulbright Foundation - Bulgaria and Fulbright Foundation - Greece for their support that allowed Victor and Galina Fet to travel and live in Bulgaria (2005) and Greece (2012). We also thank Gioele Tropea and Ersen Yaðmur for their important comments on the manuscript.

\section{References}

Birula A.A. 1900. [Scorpiones Mediterranei Musei Zoologici Mosquensis] // Izvestiya Imperatorskogo Obshchestva Lyubitelei Prirody, Istorii, Antropologii i Etnografii (Societas Caesarea Amicorum Rerum Naturalium, Anthropologiae, Ethnographiae Universitatis Moscoviensis). Vol.98. No.3(1). P.8-20 [in Russian]

Birula A.A. (Byalynitsky-Birula A.A.). 1917a. [Arachnoidea Arthrogastra Caucasica. Pars I. Scorpiones]. Mémoires du Musée du Caucase, Tiflis: Imprimerie de la Chancellerie du Comité pour la Transcaucasie, Vol.A(5), 253 pp. [in Russian]. English translation: 1964. Arthrogastric Arachnids of Caucasia. 1. Scorpions. Jerusalem: Israel Progr. Sci. Transl., 170 pp.

Birula A.A. (Byalynitsky-Birula A.A.). 1917b. [Faune de la Russie et des pays limitrophes fondée principalement sur les collections du Musée Zoologique de l'Académie des Sciences de Russie]. Arachnides (Arachnoidea). Petrograd, 1(1): xx, 227 pp. [in Russian]. English translation: 1965. Fauna of Russia and Adjacent Countries. Arachnoidea. Vol. I. Scorpions. Jerusalem: Israel Progr. Sci. Transl., xix, 154 pp.

Casale A., Giachino P.M. 2004. Hystricosphodrus vailatii, new genus and new species from Evvoia (Evia) Island, Greece (Coleoptera, Carabidae, Sphodrini) // Ann. Mus. Civ. Stor. Natur. "Giacomo Doria". Vol.96. P.341-362.

Chakrabarty P., Warren M., Page L.M., Baldwin C.C. 2013. GenSeq: An updated nomenclature and ranking for genetic sequences from type and non-type sources // ZooKeys. No.346. P.29-41.

Fet V., Soleglad M.E. 2002. Morphology analysis supports presence of more than one species in the "Euscorpius carpathicus" complex (Scorpiones: Euscorpiidae) // Euscorpius. No.3. P.1-51.

Fet V., Soleglad M.E., Parmakelis A., Kotsakiozi P., Stathi I. 2013a. A new species of Euscorpius from Tinos Island, Greece (Scorpiones: Euscorpiidae) // Rev. Ibér. Aracnol. Vol.23. P.3-10.

Fet V., Soleglad M.E., Parmakelis A., Kotsakiozi P., Stathi I. 2013b. Three more species of Euscorpius confirmed for Greece (Scorpiones: Euscorpiidae) // Euscorpius. No.165. P.1"27.

Fet V., Soleglad M.E., Zonstein S.L. 2011. The genus Akrav Levy, 2007 (Scorpiones: Akravidae) revisited // Euscorpius. No.134. P.1-49.

Gittenberger E. 1985. Speleodentorcula beroni gen. \& spec. nov. (Mollusca: Gastropoda: Orculidae) aus einer Höhle in Euboea, Griechenland // Zool. Meded. Vol. 59. No.19. P.221-228.

Gogala M., Trilar T., Drosopoulos S. 2011. Two new species and a new genus of Cicadettini (Hemiptera, Cicadidae) from the Greek island of Evia // Deutsch. Entomol. Z. Vol.58. No.1. P.105-117.

Graham M.R., Webber M.M., Blagoev G., Ivanova N., Fet V. 2012. Molecular and morphological evidence supports the elevation of Euscorpius germanus croaticus Di Caporiacco, 1950 (Scorpiones: Euscorpiidae) to Euscorpius croaticus stat. nov., a rare species from Croatia // Rev. Ibér. Aracnol. Vol.21. P.41-50.

Kinzelbach R. 1975. Die Skorpione der Ägäis. Beiträge zur Systematik, Phylogenie und Biogeographie // Zoologische Jahrbücher, Abteilung für Systematik. Bd.102. S.12-50.

Kritscher E. 1993. Ein Beitrag zur Verbreitung der Skorpione im Östlichen Mittelmeerraum // Annalen des Naturhistorischen Museums in Wien. Serie B. Botanik und Zoologie. Bd.94/95. Part B. S.377-391.

Parmakelis A., Kotsakiozi P., Stathi I., Poulikarakou S., Fet V. 2013. Hidden diversity of Euscorpius (Scorpiones: Euscorpiidae) in Greece revealed by multilocus species-delimitation approaches // Biol. J. Linn. Soc. Vol.110. P.728-748.

Parmakelis A., Pfenninger M., Spanos L., Papagiannakis G., Louis C., Mylonas M. 2005. Inference of a radiation in Mastus (Gastropoda, Pulmonata, Enidae) on the island of Crete // Evolution. Vol.59. P.991-1005.

Perreau M. 1999. Nouveaux genres et nouvelles espèces de Leptodirini (Coleoptera, Leiodidae) // Bull. Soc. Entom. France. Vol.104. No.4. P.399-406.

Rampini M., Di Russo C., Taylan M.S., Gelosa A., Cobolli M. 2012. Four new species of Dolichopoda Bolivar, 1880 from 
Southern Sporades and Western Turkey (Orthoptera, Rhaphidophoridae, Dolichopodainae) // ZooKeys. No.201. P.43-58.

Stathi I., Mylonas M. 2001. New records of scorpions from central and eastern Mediterranean area: biogeographical comments, with special reference to the Greek species // Fet V. \& Selden P.A. (eds.) Scorpions 2001. In memoriam Gary A. Polis. Burnham Beeches, Bucks: British Arachnological Society. P.287295.

Triantis K.A., Mylonas M. 2009. Greek islands, biology // Gillespie R.G., Clague D.A. (eds). Encyclopedia of Islands. Berkeley, CA, USA: University of California Press. P.388-392.

Triantis K.A., Mylonas M., Weiser M.D., Lika K., Vardinoyannis K. 2005. Species richness, environmental heterogeneity and area: a case study based on land snails in Skyros archipelago (Aegean Sea, Greece) // J. Biogeogr. Vol.32. P.1727-1735.

Trigas P., Iatrou G. 2006. The local endemic flora of Evvia (W Aegean, Greece) // Willdenowia. Vol.36. P.257-270.

Tropea G. 2013a. Reconsideration of the taxonomy of Euscorpius tergestinus (Scorpiones: Euscorpiidae) // Euscorpius. No.162. P.1-23.

Tropea G. 2013b. A new species of Euscorpius Thorell, 1876 from the western Balkans (Scorpiones: Euscorpiidae) // Euscorpius. No.174. P.1-10.

Responsible editor Yuri M. Marusik 